Case report

Perinatology Vol. 30, No. 3, September, 2019 https://doi.org/10.14734/PN.2019.30.3.175

\title{
Intraoperative Use of Resuscitative Endo- vascular Balloon Occlusion of the Aorta for Hemorrhage Control in Woman with Pla- centa Percreta Involving the Bladder
}

\section{Sung Wook Chang, MD', Ye Rim Chang, MD', Ye Jin Seon, $M D^{3}$, Jin Wan Park, MD ${ }^{3}$, Jong Soo Kim, MD ${ }^{3}$}

'Department of Thoracic and Cardiovascular Surgery, Dankook University Hospital, Cheonan; ${ }^{2}$ Department of General Surgery, Trauma Center, Dankook University Hospital, Cheonan; ${ }^{3}$ Department of Obstetrics and Gynecology, Dankook University Hospital, Cheonan, Korea

Received: 23 January 2019 Revised: 19 February 2019 Accepted: 11 March 2019

Correspondence to Jong Soo Kim, MD Department of Obstetrics and Gynecology, Dankook University Hospital, 201 Manghyang-ro, Dongnam-gu, Cheonan 31116, Korea

Tel: +82-41-550-6159

Fax: +82-41-556-0524

E-mail: soo8541@hanmail.net

Copyright@ 2019 by The Korean Society of Perinatology

This is an Open Access article distributed under the terms of the Creative Commons Attribution Non-Commercial License (http://creativecommons.org/ license/by-nc/4.0/), which permits unrestricted non-commercial use, distribution, and reproduction in any medium, provided that the original work is properly cited.
A 42-year-old woman with irregular uterine contractions and a history of two prior cesarean sections was admitted. At 35 weeks of gestation, preterm labor with vaginal bleeding led to a cesarean section. During placenta removal it was revealed to be a percreta and uncontrollable massive bleeding ensued. Intraoperative resuscitative endovascular balloon occlusion of the aorta (REBOA) was performed, which has been used as a resuscitative adjunct for trauma patients with noncompressible torso hemorrhage and a hysterectomy was successfully completed. Gauze packing for temporary abdominal closure and angio-embolization were performed to further control bleeding. The packed gauze was removed 2 days later and the patient recovered uneventfully. To the best of our knowledge, this is the first reported case in South Korean literature of the successful application of a REBOA as a salvage treatment for hemorrhage control, and REBOA could be a life-saving procedure for patients with peripartum hemorrhaging.

Key Words: Hysterectomy, Placenta, Postpartum hemorrhage, Balloon occlusion

\section{Introduction}

Placenta percreta involving the bladder is associated with torrential hemorrhaging when its removal is attempted., ${ }^{1,2}$ The possibility of hemorrhage can be minimized via proximal occlusion of the internal iliac or uterine arteries as a surgical option. ${ }^{3,4}$ However, its efficacy is limited by the rapid recruitment of an extensive collateral blood supply in the pelvis., ${ }^{2,3} \mathrm{Cur}^{-}$ rently, resuscitative endovascular balloon occlusion of the aorta (REBOA) is performed as damage control surgery for a patient with a non-compressible torso hemorrhage (NCTH), such as a massive hemorrhage originating from the abdomen or pelvis, to decrease hemorrhaging below the occlusion and increase the perfusion of the heart and brain during hemorrhagic shock. ${ }^{5,6}$ In this regard, the use of REBOA may be a life-saving procedure in patients with peripartum hemorrhage; however, the REBOA procedure has not yet been widely used to mitigate peripartum hemorrhaging due to widespread lack of knowledge and experience. In this report, we describe a patient with placenta percreta involving the bladder who was successfully treated by damage control surgery using REBOA. This is the first case in South Korea to show the important role of REBOA during hemorrhagic shock due to peripartum hemorrhage.

\section{Case}

A 42-year-old woman, gravida 2, para 2, with a history of repeat cesarean section and 
gestational age of 21 weeks, presented to the emergency department with irregular uterine contractions. The transvaginal ultrasonography showed placenta previa totalis. She was admitted for close observation and the prevention of premature labor. However, preterm labor with vaginal bleeding recurred at gestational age 35 weeks and an emergency cesarean section was decided upon.

The cesarean section was performed under spinal anesthesia and the baby was delivered safely. However, during the placenta's removal it was revealed to be a percreta, showing vascular invasion of the whole uterine myometrium and bladder, and massive pelvic bleeding ensued. After conversion to general anesthesia, a total hysterectomy was decided upon to save the patient's life. However, a total hysterectomy was impossible due to the massive and active bleeding from many collateral vessels. Since the surgical approach for aortic compression or ligation of internal iliac arteries as a life-saving procedure was impossible due to severe adhesion, urgent intraoperative consultation with the trauma surgical team was performed to complete the aortic occlusion.

A puncture using the Seldinger technique was performed on the left common femoral artery to allow for the placement of the 7 Fr Radiofocus ${ }^{\circledR}$ Introducer II (Terumo Medical Corporation, Tokyo, Japan) sheath by cardiovascular surgeon. After inserting the sheath, the 7 Fr RESCUE Balloon ${ }^{\mathrm{TM}}$ (Tokai Medical Products, Aichi, Japan) was deployed using only external landmarks. The inserted length of the balloon catheter was $25 \mathrm{~cm}$, and $10 \mathrm{~mL}$ was inflated initially without any resistance for partial occlusion of the aorta to decrease hemorrhaging below the renal arteries (Fig. 1). Balloon occlusion improved the systemic blood pressure from $60 \mathrm{mmHg}$ to $120 \mathrm{mmHg}$ and the amount of hemorrhage decreased markedly; therefore, ligation of the left internal iliac artery and the hysterectomy with division from the bladder were successful. Since the patient's hemodynamics were stable for 44 minutes after the initiation of REBOA, the balloon was deflated slowly and cautiously for 13 minutes, preventing an organ ischemia from long-term occlusion or sudden hypotension due to abrupt deflation. However, ongoing bleeding was observed in multiple sites after the deflation despite the hysterectomy, and injury between the uterus and invaded bladder was noted. Therefore, the balloon was reinflated five minutes later to $8 \mathrm{~mL}$. Oversewing the intra-abdominal pelvic bleeding sites and primary repair of the torn bladder took 29 minutes. Since ongoing bleeding was still observed after the gradual re-deflation of the balloon, the trauma surgeons conducted gauze packing using 3 QuikClot TraumaPad $^{\circledR}$ (Z-Medica LLC, Wallingford, CT, USA) and temporary abdominal closure. Packed red blood cells (53 units), fresh-frozen plasma (51 units), and platelets (41 units) were administered during the operation.

The patient was transferred immediately to the intervention room for angioembolization. Angiography showed extensive collateral vessels (Fig. 2A) and multiple bleeding foci in the pelvic cavity (Fig. 2B). The right internal iliac artery and its branches were embolized. The REBOA balloon catheter was removed in the intensive care unit after embolization. Two days later, the pelvic packs were removed without further hemorrhage and the abdominal wall was closed. On postoperative day 17 , the patient was discharged without any complications.

\section{Discussion}

Morbidly adherent placenta has increased with the cesarean delivery rate. ${ }^{1,4,7}$ Even if peripartum hysterectomy is performed

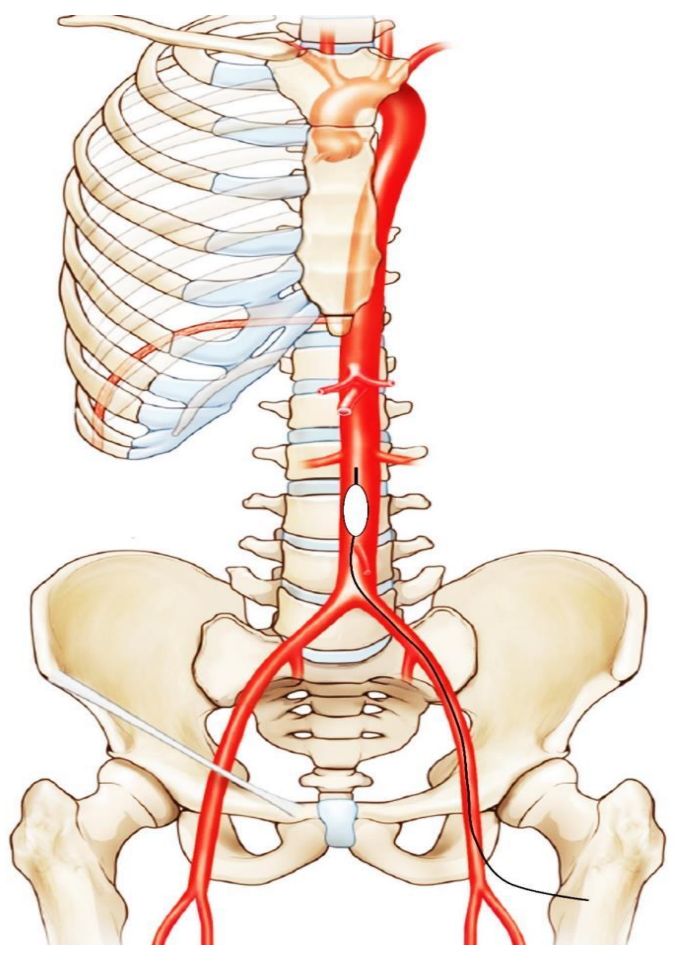

Fig. 1. Illustration of aortic occlusion shows an infrarenal resuscitative endovascular balloon occlusion of the aorta in zone 3 . 


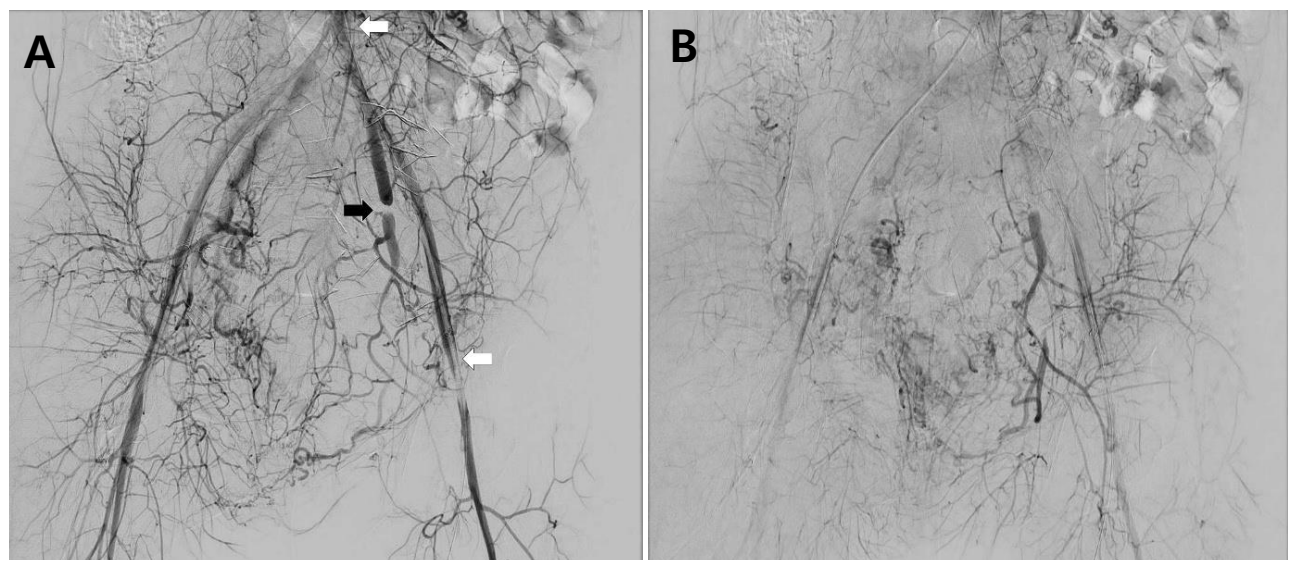

Fig. 2. (A) Angiography shows the resuscitative endovascular balloon occlusion of the aorta catheter located on lower abdominal aorta via left common femoral artery (white arrows), ligated internal iliac artery (black arrow) and many collateral vessels on pelvic cavity. (B) Multifocal extravasation despite of ligation of left internal iliac artery.

for a patient with abnormal placentation, it can result in life-threatening hemorrhaging. ${ }^{1,3,4,7}$ Among these cases, abnormally invasive placenta, including placenta percreta involving the bladder, is a major cause of maternal morbidity and mortality. ${ }^{7}$ In recent years, multidisciplinary care approaches have been accepted to reduce the rate of maternal morbidity and mortality. One involves the internal iliac artery or uterine artery balloons placed just before the time of the planned surgery to occlude the blood flow in collaboration with an interventional radiologist. ${ }^{3,4}$ However, this technique may be ineffective for controlling hemorrhaging in patients with extensive collateral vessels in the pelvis and difficult to perform in a general operating room. Furthermore, as in this case, the placenta percreta may not be diagnosed preoperatively, which would provide no time for intervention before surgery.

REBOA has increasingly been used at many centers as a resuscitative adjunct for trauma patients with life-threatening $\mathrm{NCTH}{ }^{5,6}$ REBOA helps provide time for resuscitation while preserving cerebral perfusion and coronary filling in the setting of fatal hypotension and hypovolemia secondary to hemorrhage. In patients with uncontrollable peripartum hemorrhaging, REBOA may also play a crucial role in bridging management and definitive bleeding control, which may contribute to decreased morbidity and mortality. ${ }^{2,8,9}$ According to previous literature, ${ }^{2,8-10}$ the prophylactic use of REBOA has been reported as a feasible and effective procedure for controlling hemorrhaging for a pregnant woman with abnormal placentation undergoing cesarean delivery. However, the use of a REBOA procedure for peripartum hemorr- haging has not yet been performed in South Korea due to lack of experience.

Performing the REBOA procedure intraoperatively requires keeping several important points in mind to maximize its effect and minimize complications. First, there may be several procedure-related complications following REBOA, such as failed arterial access and vascular injuries. ${ }^{6,11}$ In an effort to decrease procedure-related complications, ultrasonography-guided percutaneous arterial puncture is generally recommended. ${ }^{11}$ Smaller sheaths and balloon catheters such as of 7 Fr size are also recommended. ${ }^{12}$ In this case, an arterial puncture in the common femoral artery with a $7 \mathrm{Fr}$ sheath was performed without complication approximately $2 \mathrm{~cm}$ below the inguinal ligament since ultrasonography was not instantly available.

Second, perfusion-related complications such as organ ischemia may occur as a consequence of aortic occlusion. To minimize perfusion-related ischemic complication during aortic occlusion, real-time fluoroscopic guidance using a C-arm and portable serial X-ray is recommended for accurate balloon placement. However, when radiologic equipment is unavailable, the umbilicus as an anatomic marker and the usual length of the segment can be used to estimate the balloon position. ${ }^{13}$ Since radiologic equipment was not readily available in this case, the anatomic marker was used for the estimation, and the balloon was inflated to $25 \mathrm{~cm}$ long without complication.

Third, partial REBOA (pREBOA) and intermittent REBOA (iREBOA) can be adopted to decrease ischemic events. ${ }^{14}$ There 
should be no resistance during balloon inflation in the pREBOA status, and incomplete occlusion of aorta can balance minimizing ongoing bleeding and lessening distal ischemia. In iREBOA status, the balloon is intermittently inflated and deflated to prevent organ ischemia. However, appropriate volumes and times for pREBOA and iREBOA still remain to be investigated. Qasim et al. ${ }^{15}$ reported that the ideal time for aortic occlusion was no longer than 40 minutes to prevent ischemic complications. However, based on recent reports, pREBOA and/or iREBOA times may potentially extend the occlusion duration beyond the previously reported ideal occlusion time. ${ }^{15}$ In such a case, $10 \mathrm{~mL}$ was inflated without any resistance to achieve pREBOA, with an overall duration of 82 minutes. In addition, intermittent de-ballooning was conducted for five minutes and the patient had no complications.

In conclusion, REBOA could be a life-saving procedure in patients with abnormal placentation associated with torrential hemorrhaging. Provided that several considerations detailed in this paper are kept in mind, REBOA may be a feasible and safe procedure to save patients with peripartum hemorrhage. Even if REBOA cannot be easily performed by an obstetric surgeon, a multidisciplinary approach may facilitate its utilization. However, the effects of REBOA on next pregnancy and its long term prognosis are still not identified. Consequently, there is a very real need for further studies and establishing the indications for REBOA for postpartum hemorrhage.

\section{References}

1) Wu S, Kocherginsky M, Hibbard JU. Abnormal placentation: twentyyear analysis. Am J Obstet Gynecol 2005;192:1458-61.

2) Parra MW, Ordonez CA, Herrera-Escobar JP, Gonzalez-Garcia A, Guben J. Resuscitative endovascular balloon occlusion of the aorta for placenta percreta/previa. J Trauma Acute Care Surg 2018;84:403-5.

3) Cali G, Forlani F, Giambanco L, Amico ML, Vallone M, Puccio G, et al. Prophylactic use of intravascular balloon catheters in women with placenta accreta, increta and percreta. Eur J Obstet Gynecol Reprod Biol 2014;179:36-41.

4) Ballas J, Hull AD, Saenz C, Warshak CR, Roberts $A C$, Resnik RR, et al.
Preoperative intravascular balloon catheters and surgical outcomes in pregnancies complicated by placenta accreta: a management paradox. Am J Obstet Gynecol 2012;207:216.e1-5.

5) Stannard A, Eliason JL, Rasmussen TE. Resuscitative endovascular balloon occlusion of the aorta (REBOA) as an adjunct for hemorrhagic shock. J Trauma 2011;71:1869-72.

6) Kim DH, Chang SW, Matsumoto J. The utilization of resuscitative endo vascular balloon occlusion of the aorta: preparation, technique, and the implementation of a novel approach to stabilizing hemorrhage. J Thorac Dis 2018;10:5550-9.

7) Brennan DJ, Schulze B, Chetty N, Crandon A, Petersen SG, Gardener G, et al. Surgical management of abnormally invasive placenta: a retrospec tive cohort study demonstrating the benefits of a standardized opera tive approach. Acta Obstet Gynecol Scand 2015;94:1380-6.

8) Manzano-Nunez R, Escobar-Vidarte MF, Naranjo MP, Rodriguez F, Ferrada P, Casallas JD, et al. Expanding the field of acute care surgery: a systematic review of the use of resuscitative endovascular balloon occlusion of the aorta (REBOA) in cases of morbidly adherent placenta. Eur J Trauma Emerg Surg 2018;44:519-26.

9) Ordonez CA, Manzano-Nunez R, Parra MW, Rasmussen TE, Nieto AJ, Herrera-Escobar JP, et al. Prophylactic use of resuscitative endovascular balloon occlusion of the aorta in women with abnormal placentation: a systematic review, meta-analysis, and case series. J Trauma Acute Care Surg 2018;84:809-18.

10) Wu Q, Liu Z, Zhao X, Liu C, Wang Y, Chu Q, et al. Outcome of pregnancies after balloon occlusion of the infrarenal abdominal aorta during caesarean in 230 patients with placenta praevia accreta. Cardiovasc Intervent Radiol 2016;39:1573-9.

11) Ogura T, Lefor AK, Nakamura M, Fujizuka K, Shiroto K, Nakano M. Ultra sound-guided resuscitative endovascular balloon occlusion of the aorta in the resuscitation area. J Emerg Med 2017;52:715-22.

12) Matsumura $Y$, Matsumoto J, Kondo $H$, Idoguchi $K$, Ishida T, Kon $Y$, et al. Fewer REBOA complications with smaller devices and partial occlusion: evidence from a multicentre registry in Japan. Emerg Med J 2017;34: 793-9.

13) Pezy P, Flaris AN, Prat NJ, Cotton F, Lundberg PW, Caillot JL, et al. Fixeddistance model for balloon placement during fluoroscopy-free resuscita tive endovascular balloon occlusion of the aorta in a civilian population. JAMA Surg 2017;152:351-8.

14) Johnson MA, Neff LP, Williams TK, DuBose JJ, EVAC Study Group. Partial resuscitative balloon occlusion of the aorta (P-REBOA): clinical technique and rationale. J Trauma Acute Care Surg 2016;81 (5 Suppl 2 Proceedings of the 2015 Military Health System Research Symposium):S133-7.

15) Qasim Z, Brenner M, Menaker J, Scalea T. Resuscitative endovascular balloon occlusion of the aorta. Resuscitation 2015;96:275-9. 